\title{
Micro-Electric Propulsion (EP) Solutions for Small
}

\section{Satellite Missions}

\author{
V. J. Lappas ${ }^{1}$, S. J. Pottinger ${ }^{1}$, A. K. Knoll ${ }^{1}$, P. V. Shaw ${ }^{1}$, D. Lamprou ${ }^{1}$, T. Harle, B. Melly, M. Perren ${ }^{2}$ \\ ${ }^{1}$ Surrey Space Centre, University of Surrey \\ Guildford, United Kingdom GU2 7XH \\ v.lappasesurrey.ac.uk \\ s.pottingeresurrey.ac.uk \\ a.knollesurrey.ac.uk \\ ${ }^{2}$ EADS Astrium, Rue Laurent Pichat \\ 75216 Paris Cedex 16, France \\ Matthew.PERRENQastrium.eads
}

\begin{abstract}
Electric propulsion (EP) technologies offer performance advantages compared to chemical systems. The implementation of electric thrusters in small spacecraft has been prohibited by the high power demands of conventional thrusters and the complexity of the required power conditioning units and propellant supply system. Advances in micro electronics have made the use of EP on small satellites feasible. Research and development on 3 technologies: 1. Helicon Double Layer Thruster (HDLT), 2. Hollow Cathode Thruster (HCT) and 3. Pulsed Plasma Thruster (PPT) has focussed on characterisation and design optimisation for small, micro and nanosatellite missions respectively. All thrusters operate in differing regimes and display varying degrees of development. Initial characterisation of the HDLT operating with argon propellant shows a maximum measured thrust of $1.7 \mathrm{mN}$ for an operating power of $500 \mathrm{~W}$. The current investigation has demonstrated a thrust of $1.7 \mathrm{mN}$ and specific impulse of 70s at 50W for a HCT operating with krypton propellant. The development of PPTs for the STRaND-1 nanosatellite mission demonstrates the option of utilising EP to extend the lifetime of CubeSat missions.

Index Terms-Electric Propulsion
\end{abstract}

\section{INTRODUCTION}

The space sector has been reluctant to widely implement Electric Propulsion on board commercial satellites. This is due to the relative complexity of the technologies and the incumbent power consumption trading off against the high efficiency of the thrusters. Proliferation of research and development of satellite technologies from government organisations to academia and private enterprise has resulted in a focus on low cost technologies with a rapid design and development phase. While advances in microelectronics have allowed the mass, volume and cost of power supply units for EP systems to be reduced [1]. There now exists a wide variety of EP options with varying levels of complexity and technology readiness levels (TRL) which can be exploited to provide options for station keeping, orbit maintenance and orbit raising.
This paper reports the research and development progress at the Surrey Space Centre (SSC) of three different propulsion systems, two of which have been selected for flight missions. The Helicon Double Layer Thruster (HDLT) is a radio frequency (RF) driven device that has been demonstrated to operate over input powers of hundreds of Watts to kilo Watts. For the purposes of small satellite applications operating powers up to $500 \mathrm{~W}$ have been investigated. The Hollow Cathode Thruster (HCT) operates using a direct current discharge at maximum power levels of $100 \mathrm{~W}$. This technology in the near term has been identified as a potential thruster for use on microsatellite missions with a long term application on commercial Geostationary (GEO) satellites. The Pulsed Plasma Thruster (PPT) produces a pulsed discharge with a duration of approximately $5 \mu$ s with a discharge frequency of $0.17 \mathrm{~Hz}$. Each thruster module requires a maximum operating power of less than $1.5 \mathrm{~W}$. This technology has been developed to provide propulsion options for CubeSats and will be flown on the STRaND-1 mission, which is a collaboration between the Surrey Space Centre and Surrey Satellite Technology Limited.

The objective of current research and development efforts is to increase the TRL of the EP technologies investigated through comprehensive performance characterisation in a laboratory environment combined with proof of in flight operation on technology demonstration missions.

\section{Micro-Electric Propulsion Development}

The propulsion systems presented below were developed and characterised within the Surrey Space Centre propulsion test facility. Evaluation of thruster operation and performance was undertaken in a space simulation chamber with dimensions of $1.2 \mathrm{~m}$ diameter and $1.2 \mathrm{~m}$ length. Vacuum chamber pressures of the order of $10^{-6}$ mbar may be achieved without load. For cases where thrust measurements are shown the data was obtained using an in house developed pendulum type thrust stand, described in detail in 
Reference [2]. The following sections describe the EP systems under investigation and present relevant performance data.

\subsection{Helicon Double Layer Thruster}

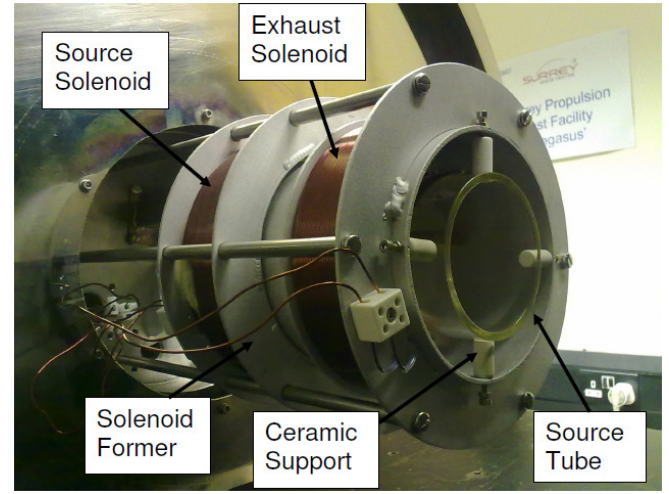

Fig. 1. The Helicon Double Layer Thruster mounted in the vacuum propulsion test facility

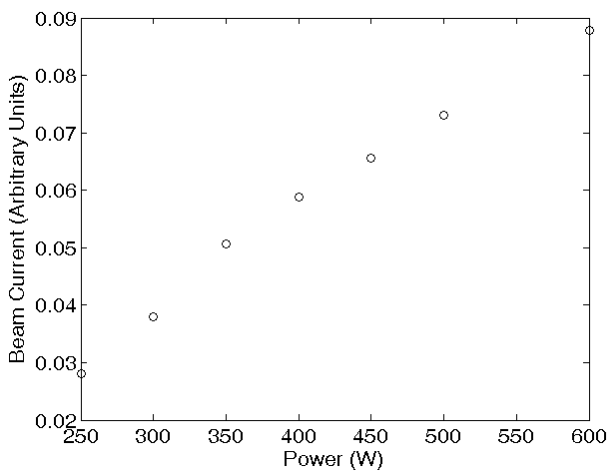

Fig. 2. Collected beam current measured with an RFEA placed on the thrusters' central axis $5 \mathrm{~cm}$ downstream of the source exit

The Helicon Double Layer Thruster, shown in Figure 1, generates plasma using a helicon source and accelerates a high energy population of ions across a double layer $[3,4]$. The thruster offers advantages compared to conventional EP technologies due to its simple design and inherent ion acceleration mechanism. Ions are accelerated by a combination of the potential drop across the double layer and detachment from a diverging magnetic field [2]. As a result the observed ion energies in the exhaust beam are orders of magnitude lower than those of ion thrusters or Hall Effect thrusters. The generated exhaust beam is also macroscopically neutral. Therefore, interactions with spacecraft surfaces are reduced without the need of an external neutraliser and sputter damage caused by the impact of high energy ions is eliminated. The comparatively low energy of the ions results in reduced thruster channel erosion which supports long operational life. The reduced number of complex components in the thruster design decreases the number of wear mechanisms that may precipitate failures and end of life. The HDLT is also able to operate using a variety of propellants. The thruster has the potential to act as a mission enabling technology for long duration flight missions. For these reasons the HDLT has been the subject of recent research and development efforts.

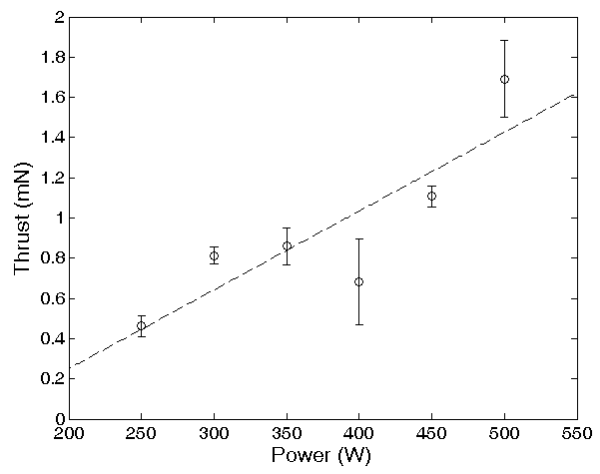

Fig. 3. Thrust generated as a function of power for the HDLT operating on $32 \mathrm{sccm}$ of argon

The performance of a non optimised laboratory model HDLT has been investigated using direct thrust measurements and a retarding field energy analyser (RFEA), described in detail in Reference [5]. An RFEA placed on the central axis of the HDLT $50 \mathrm{~mm}$ downstream of the source tube exit was used to record plasma characteristics for the thruster operating with argon propellant over an input power range of $250 \mathrm{~W}$ to $600 \mathrm{~W}$. All data was recorded with the HDLT utilising the exhaust solenoid only, a peak diverging magnetic field strength of $100 \mathrm{G}$ was applied corresponding to a current of $3.5 \mathrm{~A}$. A Pyrex source tube with an outer diameter of $80 \mathrm{~mm}$ and inner diameter of $70 \mathrm{~mm}$ was used in all cases. Figure 2 shows the measured beam current which represents the high energy ion population. The data shows that the plasma beam current increases with increasing power demonstrating improved thruster performance. Direct thrust measurements were recorded at $50 \mathrm{~W}$ increments from $250 \mathrm{~W}$ to $500 \mathrm{~W}$ and show a maximum thrust of $1.7 \pm 0.2$ $\mathrm{mN}$ obtained for an RF input power of $500 \mathrm{~W}$ with a propellant flow rate of $32 \mathrm{sccm}$ of argon $(1 \mathrm{mg} / \mathrm{s})$, see Fig. 3 . Both the beam current and thrust increase linearly with increasing input RF power. The initial data from a non optimised device indicates that further development is required in order to achieve the performance necessary for ambitious flight applications. Future work will focus on enhancing performance and increasing the TRL of the thruster.

\subsection{Hollow Cathode Thruster}

The Hollow Cathode Thruster (HCT) is a conventional hollow cathode used in a standalone configuration to generate thrust. An insert impregnated with earth metal oxides is heated to approximately $1100^{\circ} \mathrm{C}$ which results in electron emission. The liberated electrons interact with the propellant gas generating ions, a sheath forms at the insert surface resulting in enhanced electron emission. When a sufficient ion density is achieved a discharge is initiated between the cathode and keeper electrode due to the application of voltage at the keeper electrode. 
The operation of hollow cathode technologies has been studied extensively for applications as electron sources, beam neutralizers and plasma contactors. However, an understanding of the detailed physics of the operation of the device remains elusive particularly for the process of thrust generation which now has several proposed theories [6-9].

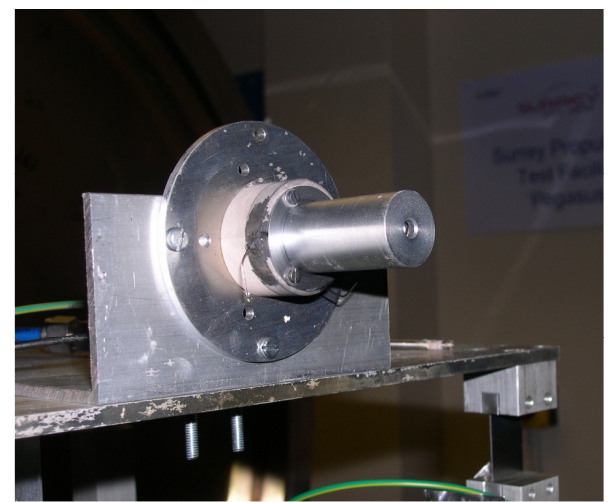

Fig. 4. The HCT mounted on the inverted pendulum thrust stand

There has been interest in developing all electric GEO satellites for telecommunications applications [10]. It has been proposed that the use of HCTs for north south, east west station keeping and wheel off-loading provide propellant mass savings. An alternative niche application is for the HCT is to act as the main propulsion system for low Earth orbit microsatellites with the capability to produce specific impulse of approximately $200 \mathrm{~s}$ compared to $50 \mathrm{~s}$ of a typical low power resistojet [11].

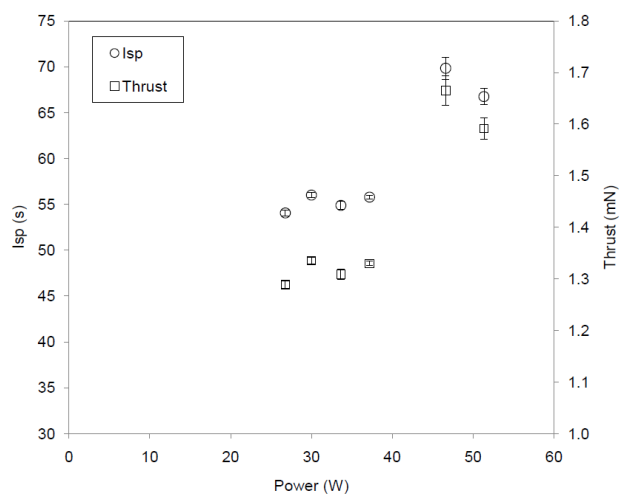

Fig. 5. Isp and thrust generated as a function of power for the HCT operating on $39 \mathrm{sccm}$ of krypton

The current research and development effort aims to develop a flight worthy HCT, supporting power and control electronics along with a simple propellant supply system for microsatellite applications. Research is focussed on the identification of the thrust generation mechanism in order to optimise thruster performance. Initial characterisation of an in house developed HCT operating in an enclosed keeper configuration without the use of an external anode (see Fig. 4) shows increasing thrust with increasing discharge power, as expected. The data presented in Fig. 5 are direct thrust measurements for the HCT operating with an applied propellant flow rate of $39 \mathrm{sccm}$ of krypton $(\sim 2.5 \mathrm{mg} / \mathrm{s})$. For this investigation the keeper to orifice distance is $1 \mathrm{~mm}$, keeper bore diameter $4 \mathrm{~mm}$ and orifice bore $1 \mathrm{~mm}$. Power levels up to $50 \mathrm{~W}$ have been presented with further testing at higher power levels and using xenon propellant commencing in the near future in order to achieve target performance of $150-200$ s specific impulse and comply with the flight proven heritage of xenon resistojet systems.

\subsection{Pulsed Plasma Thruster}

Pulsed Plasma Thrusters offer an ideal propulsion solution for satellite missions ranging from small satellites to CubeSats due to the simplicity and scalability of the thruster $[12,13]$. The technology has an extensive flight heritage and research base which may be exploited to develop systems with the required performance and design attributes necessary for niche applications. Current work at the Surrey Space Centre aims to develop and launch the first electric propulsion system to successfully operate on a CubeSat. The STRaND-1 (Surrey Technology Research and Nanosatellite Development) satellite is a collaborative mission between SSC and Surrey Satellite Technology Ltd. (SSTL) where 8 PPTs will be integrated into a three unit CubeSat with dimensions $10 \mathrm{~cm} \times 10 \mathrm{~cm} \times 30 \mathrm{~cm}$. The thrusters will be mounted on three separate PC 104 boards (See Fig. 6) and each will produce an estimated impulse bit of $\mathrm{I}_{\mathrm{Bit}}=0.6 \mu \mathrm{Ns}$. The total operating power draw by the system (thruster and supporting electronics) is approximately $6 \mathrm{~W}$.

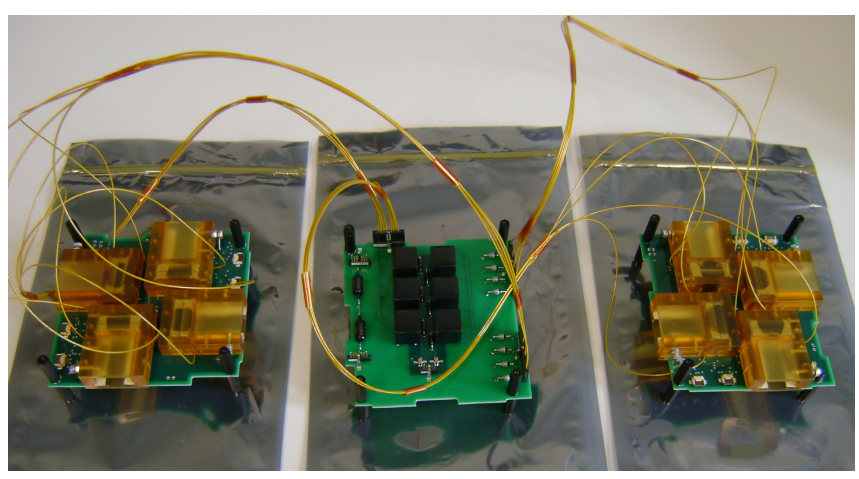

Fig. 6. STRaND-1 PPT module

The performance of the PPT scales with the energy supplied to the thruster as it pulses. The pulsed nature of the device is the reason behind its low power consumption. The size and volume of the capacitor that is used to store the energy between discharges dictates the eventual performance of the thruster. In the case of the developed $\mu$ PPT for STRaND-1 $0.86 \mathrm{~J}$ is stored and the thruster is predicted to have a specific impulse of approximately $321 \mathrm{~s}$.

The STRaND-1 CubeSat is a technology demonstrator mission which includes state of the art technology including a smart phone. In the case of the developed PPTs the emphasis is placed on gaining flight heritage and evaluating the performance of the module with flight data. The PPTs 
will be used for precision control and pointing, momentum dumping and lifetime extension.

The propulsion module used for STRaND-1 has the potential to be scaled up for operation on a $100 \mathrm{~kg}$ satellite, the mass and volume gained compared to alternative thruster systems would allow for larger discharge capacitors to be used which would result in increased thruster performance. This would allow the thruster to be used as a main propulsion system for both orbit and attitude control manoeuvres

\section{CONCLUSIONS AND FUTURE WORK}

Three EP technologies have been developed for applications on small, micro and nanosatellites. Direct thrust measurements performed on a laboratory prototype HDLT and HCT show thrust levels in the range of 1 to $2 \mathrm{mN}$. Future work on HDLT development will focus on performance enhancement and thruster design using flight compliant materials and components. The HCT displays a more advanced level of development compared to the HDLT with flight ready components and electronics. The future exploitation of this technology requires successful operation in space with lessons learnt from flight missions providing further design enhancements. If successful, the STRaND-1 mission will be the first CubeSat to incorporate electric propulsion on this class of satellite. The thruster developed will provide complete attitude control for the 3 unit satellite.

\section{ACKNOWLEDGMENT}

The HDLT project is funded through the University of Surrey's strategic partnership agreement with EADS Astrium.

\section{REFERENCES}

[1] L. C. Croci, P. Galantini, and C. Marana, "Influence of ion propulsion on the electrical power system sizing," Second European Spacecraft Propulsion Conference, pp. 619-625, 1997

[2] S. J. Pottinger, V. J. Lappas, C. Charles and R. W. Boswell, "Performance Characterization of a Helicon Double Layer Thruster using Direct Thrust Measurements," J. Phys. D: Appl. Phys. 44 (2011) 235201 (5pp)

[3] C. Charles "Plasmas for Spacecraft Propulsion," J. Phys. D: Appl. Phys. 42 (2009) 163001 (pp. 18)

[4] M. D. West, C. Charles and R. W. Boswell, "Testing a Helicon Double Layer Thruster Immersed in a Space-Simulation Chamber," Journal of Propulsion and Power, vol. 24, pp. 134-141, 2008

[5] D. L. Rudakov, M. G. Shats, C. Charles, R. W. Boswell and J. Howard, "Overview of probe diagnostics on the H1 heliac," Rev. Sci. Instrum., vol.70, pp. 476-479, 1999

[6] A. Snyder and B. A. Banks, "Thrust measurements of a hollow cathode discharge," NASA Technical Note, vol. NASA TN D- 6705, pp. 1-21, 1972.

[7] A. N. Grubisic and S. B. Gabriel, "Understanding hollow cathode thrust production mechanisms," $31^{\text {st }}$ International Electric Propulsion Conference, Ann Arbor, USA, pp 1-27, 2009

[8] I. G. Mikellides, I. Katz, D. M. Goebel, and J. E. Polk, "Hollow cathode theory and experiment II. A two-dimensional theoretical model of the emitter region," Journal of Applied Physics, vol. 98, pp. $1-14,2005$.

[9] D. Goebel, K. K. Jameson, I. Katz, and I. G. Mikellides, "Potential fluctuations and energetic ion production in hollow cathode discharges," Physics of Plasmas, vol. 14, pp. 1-15, 2007.

[10] M. H. Kaplan, "All Electric Thruster Control of a Geostationary Communications Satellite," Journal of Spacecraft and Rockets, vol. 20, pp. 119-125, 1973

[11] M. Pollard, "The Design, Build, Test and In-orbit Performance of Low cost Xenon Warm Gas Propulsion Systems," Space Propulsion 2010, SP2010_1839886.

[12] M. Martinez-Sanchez, and J. E. Pollard, "Spacecraft Electric Propulsion--An Overview," Journal of Propulsion and Power, vol. 14, pp. $1167-1179,1998$

[13] R.L. Burton, P.J. Turchi, "Pulsed plasma thruster," Journal of Propulsion and Power, vol. 14 (5) pp. 716-735, 1998 